\title{
Strategies for Inclusion of Structural Mass Estimates in the Direct-Drive Generator Optimization Process
}

\author{
Matthew L. Henriksen and Bogi B. Jensen \\ Center for Electrical Power and Energy \\ Technical University of Denmark \\ 2800 Kongens Lyngby, Denmark
}

\begin{abstract}
Usage of a lookup table containing the structural mass and air gap deformation for direct-drive wind turbines of various dimensions is demonstrated. The development of the table is described in detail. Optimal generator designs while both neglecting and considering the structural mass are also included.
\end{abstract}

\section{Introduction}

In comparing various wind turbine drivetrain topologies, optimal design should be strongly considered in order to encourage a fair comparison. For wind turbines this is a challenging task, due in large part to huge fundamental differences between the geared and direct-drive concepts, which are both competitive options in today's marketplace. Many designers of electrical machines have delved into this topic, and it has been made clear that the comparison of direct-drive and geared drivetrains must take some mechanical design aspects into consideration [1], [2], [3].

For the geared concepts, this means that the mass, cost, losses, and if possible the reliability of the gearbox should be taken into the comparison. When it comes to direct-drive wind turbines, their unique feature is a need for significant structural mass, which has the purpose of preventing the air gap from deforming.

The required structural mass can be estimated by using mechanical finite element analysis (FEA). While the prospect of using multiphysics simulations to consider the electromagnetic performance of the generator at the same time as the mechanical deformation exists, it would result in a very complex simulation, both in terms of configuration and in scope.

Another idea, investigated in this paper, is performing the structural mass simulations ahead of time and storing the results [4]. In this paper, some background is provided in order to explain the desired range of the lookup table. Next, the employed structural configuration and parametric study is explained. Finally, results for the estimated cost of energy are demonstrated while using the lookup table, and also while ignoring the need for structural mass.

\section{Design of the Lookup Table}

In this section the basics of direct-drive wind turbine design are introduced, in order to demonstrate the need for consideration of the structural mass. Then, the factors which will influence the air gap deformation are introduced, and the organization of the structural mass lookup table is demonstrated.

\subsection{Direct-Drive Design}

The diameter of a multi-megawatt wind turbine generator will be several meters, as can be conceptually understood with reference to equation (1) giving the maximum electromagnetic torque [5]:

$$
T_{\max }=\sqrt{2} V A \hat{B}
$$

The electrical loading $A$ describes the number of ampere turns per meter of air gap circumference. The electrical loading is dependent on the method used to cool the machine, and assuming a forced-air system a reasonable upper limit is $80 \mathrm{kA} / \mathrm{m}$. The magnitude of the fundamental component of air gap flux density, $\hat{B}$, is limited by the available magnets, as well as the saturation of the stator core. With modern rare earth magnets, the upper limit for the peak air gap flux density is $1.0-1.2 \mathrm{~T}$. Considering that a $15 \mathrm{rpm}$ wind turbine would require around $2 \mathrm{MNm}$ to drive a $3 \mathrm{MW}$ generator, the interior volume $V$ for a highly loaded $3 \mathrm{MW}$ machine should approach $20 \mathrm{~m}^{3}$.

The need for a physically large generator should be clear. For a required volume, the total mass can be reduced if the machine takes on a pancake shape. That is, the diameter becomes large rather than the length becoming large. At the same time, the usage of a high number of poles means that only a very low coreback thickness is required.

An attractive stress $q$ between the rotor and stator occurs, proportional to the air gap flux density, squared:

$$
q=\frac{1}{2} \frac{\hat{B}^{2}}{\mu_{0}}
$$

The stiffness in the air gap for most electrical machines is provided in part by a sizable coreback, but for the case of a direct-drive machine the coreback has no need to be so thick. Thus, the need for dedicated, inactive structural support mass arises. 


\subsection{Support Structure}

The support structure assumed for the stator can be seen in Figure 1. The structure is a hollow drum, with one disc on either side of its length. It also has a rim, with length in the axial direction and width in the radial direction. The rim acts somewhat as an extension of the coreback thickness. The discs on either side provide a connecting point between the generator's stator and mounting points inside the nacelle. The stator support mass can be in this was by approximated by the mass of two radial discs and one cylindrical rim.

The rotor will be on the outside of the machine. The rotor structure resembles a drum as well, only with one of the ends removed. Accordingly, the rotor structural mass can be estimated as the mass of one cylindrical rim and one radial disc.

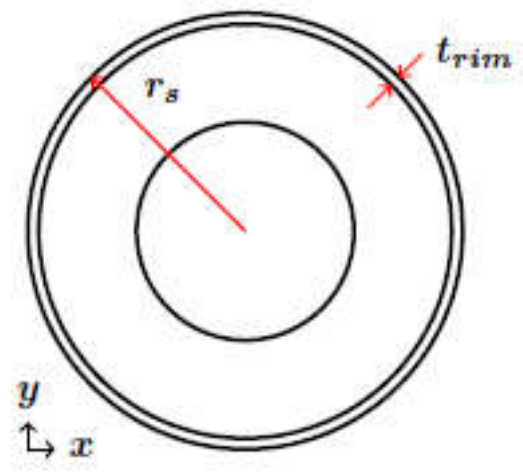

(a)

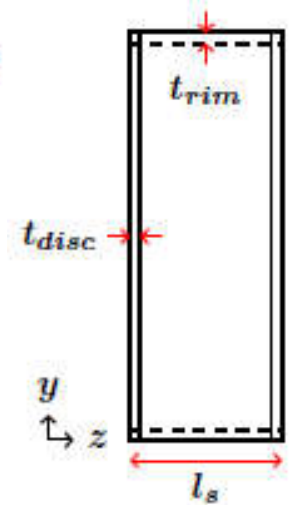

(b)
Figure 1: Views of the (a) radial plane and (b) axial plane of the stator support structure

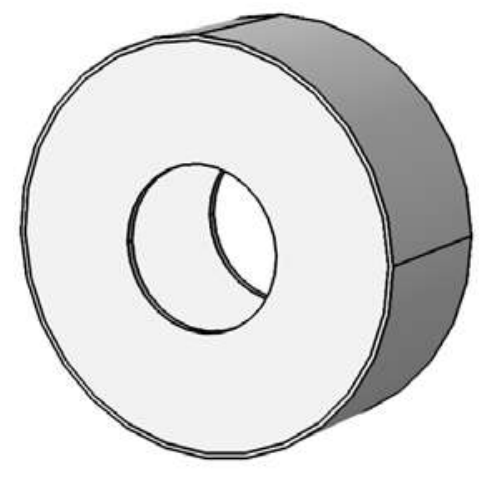

(a)

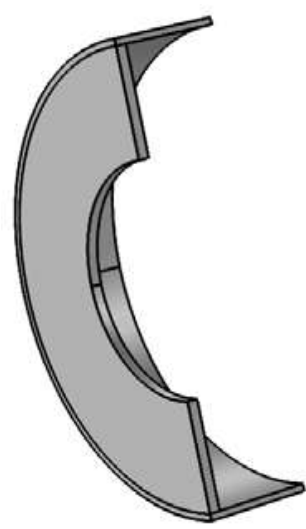

(b)
Figure 2: 3D visualization of the (a) stator and (b) rotor support structures

\subsection{Bounds for the Lookup Table}

The deformation in the air gap, as well as the required structural mass to keep this to an acceptable level, will be influenced by several factors:
- $\quad$ The peak air gap flux density $\hat{B}[\mathrm{~T}]$

- The air gap radius $r_{s}[\mathrm{~m}]$

- The length of the machine $l_{s}[\mathrm{~m}]$

- Structural rim thickness $t_{r i m}[\mathrm{~m}]$

- $\quad$ Structural disc thickness $t_{\text {disc }}[\mathrm{m}]$

The lookup table should therefore hold structural mass and deformation results for combinations of these five factors. The initial region of interest is described in Table 1 . The possibility for high and low flux densities is included, as well as many combinations of length and radius. For every combination or radius and length, all flux densities are considered. For every flux density considered, all combinations of disc and rim thickness are assessed.

Table 1: The original bounds for the lookup table

\begin{tabular}{llllll}
\hline & $\hat{B}[\mathrm{~T}]$ & $r_{s}[\mathrm{~m}]$ & $l_{s}[\mathrm{~m}]$ & $t_{\text {rim }}[\mathrm{m}]$ & $t_{\text {disc }}[\mathrm{m}]$ \\
\hline Min & 0.5 & 2.0 & 1.0 & 0.04 & 0.04 \\
Step & 0.1 & 0.1 & 0.1 & 0.02 & 0.02 \\
Max & 1.2 & 3.0 & 2.5 & 0.1 & 0.1 \\
\hline
\end{tabular}

\section{The Parameterized Structural Study}

The method for gathering all data for completion of the lookup table is detailed in this section. In short, a parametric simulation campaign was initiated. Figure 2 shows examples of the structures which were analyzed using the Structural Mechanics physics package in COMSOL. It was possible to use symmetry to reduce the number of mesh elements, so the simulation models were actually more similar to that shown in Figure 2 (b), which shows half of the rotor.

\subsection{Study Configuration and Limitations}

Several sources of air gap deforming stresses exist in direct-drive wind turbines. The largest of these is the normal component of Maxwell's stress tensor, and this is the only item under investigation in this work [1]. Air gap deformation due to thermal expansion is neglected, as is deformation due to gravity.

The rotor and stator are simulated separately. This is a useful measure, because some of the configurations of interest may otherwise result in collision of the rotor and stator which would cause the study to crash.

Taking the stator as the example, Figure 3 shows the boundary conditions used during the simulations. Fixed boundary conditions are applied at the inner surfaces of the support discs. These boundary conditions represent a strong fixture by which the stator support structure is held in place. A boundary load is applied to the outer surface of the stator, representing the attraction between the rotor and stator. For the rotor, a boundary load of equal magnitude and opposite direction is applied to the inner surface. There is only one support disc for the rotor structure, but a similar fixed boundary condition is applied to its inner surface. 
An additional effect not included is secondary deformation. In reality, once the initial deformation takes place, a reduction in the air gap reluctance will occur. This reduction in reluctance can lead to increased air gap flux density, and further deformation [4].

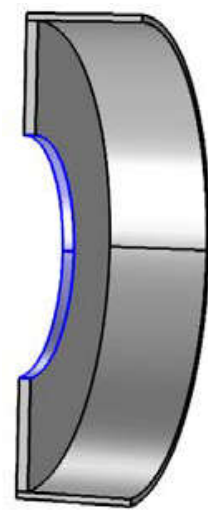

(a)

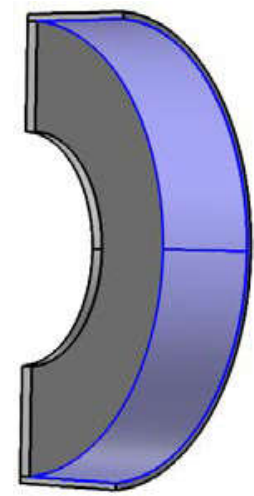

(b)
Figure 3: Boundary conditions for simulation of the rotor: (a) fixed and (b) boundary load

\subsection{Example Simulations}

To show the effect of the structural mass on the deformation of the air gap, two examples will be demonstrated. The details of the two examples can be seen in Table 2.

Both examples use a rotor with the same dimensions, and are subject to the same peak air gap flux density. The difference between the two models is in the structural support: example (b) uses a thicker support disc than is used in example (a).

The deformation resulting from these simulation parameters can be seen in Figure 4. The support structure used in example (a) allowed the air gap to deform by about $0.35 \mathrm{~mm}$, while for the second example this was reduced to nearly $0.25 \mathrm{~mm}$. Example (a) utilized a total of $8.3 \mathrm{t}$ of structural steel. In example (b) that value rose to $11.8 \mathrm{t}$

Table 2: Parameters for two simulations of the rotor support structure

\begin{tabular}{llllll}
\hline & $\hat{B}[\mathrm{~T}]$ & $r_{s}[\mathrm{~m}]$ & $l[\mathrm{~m}]$ & $h_{\text {rim }}[\mathrm{m}]$ & $t_{\text {disc }}[\mathrm{m}]$ \\
\hline (a) & 0.9 & 2.0 & 1.0 & 0.06 & 0.05 \\
(b) & 0.9 & 2.0 & 1.0 & 0.06 & 0.10 \\
\hline
\end{tabular}

\section{Usage of Lookup Table in Generator Design Process}

In this section the incorporation of the lookup table into the optimal design process is explained, and example results are provided.

\subsection{Design Process}

The machine design process utilizes finite element analysis to assess electromagnetic losses, torque production, and the peak air gap flux density for each candidate design. The air gap flux density, along with
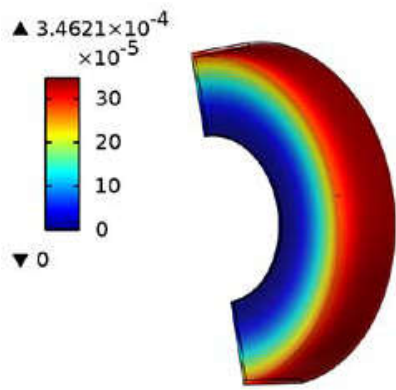

(a)
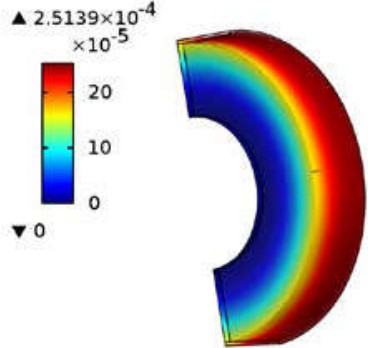

(b)

Figure 4: Two example results from simulation of the rotor deformation, indicating the magnitude of the deformation [m].

the radius and length for the machine, are used to determine the required structural mass. It is important to state the allowed deformation $\delta_{\max }$ of the air gap. The value currently being used in this work is $15 \%$ of the air gap length:

$$
\delta_{\max }=0.15 * l_{g}
$$

The allowed deformation is divided evenly between the rotor and the stator, that is, if the air gap is allowed to deform by $1 \mathrm{~mm}$, then the rotor and stator are both allowed to deform by $0.5 \mathrm{~mm}$.

For each combination of flux density, length, and diameter of the machine, the deformation and total mass of 16 structural configurations have been examined and stored in the lookup table. The configuration which is selected for each candidate design is the lightest one which meets the deformation requirement. The cost of the structure, assumed to be directly proportional to the mass, is then taken into account along with the active mass of the generator.

\subsection{Trends in the Required Structural Mass}

To briefly demonstrate the response of the required structural mass to the design variables, Figure 5 shows the masses of the lightest acceptable rotor structures for the condition that the air gap is allowed to deform by $10.0 \%$. The influences of the machine length and air gap 
flux density are apparent. Both of these variables show strong, positive correlation with the structural mass.

The effect of the radius is a little more difficult to isolate. Clearly, increasing the radius of two identical structures described by the thickness of the structural rim and discs, will tend to increase their mass. However, as the radius increases, the length of the air gap increases as well. Along with this, the allowed deformation of the air gap, in meters, will increase as indicated by equation (3). It follows then that the rim and discs could be made thinner, and in this way there is an additional relationship between the radius and the structural mass.
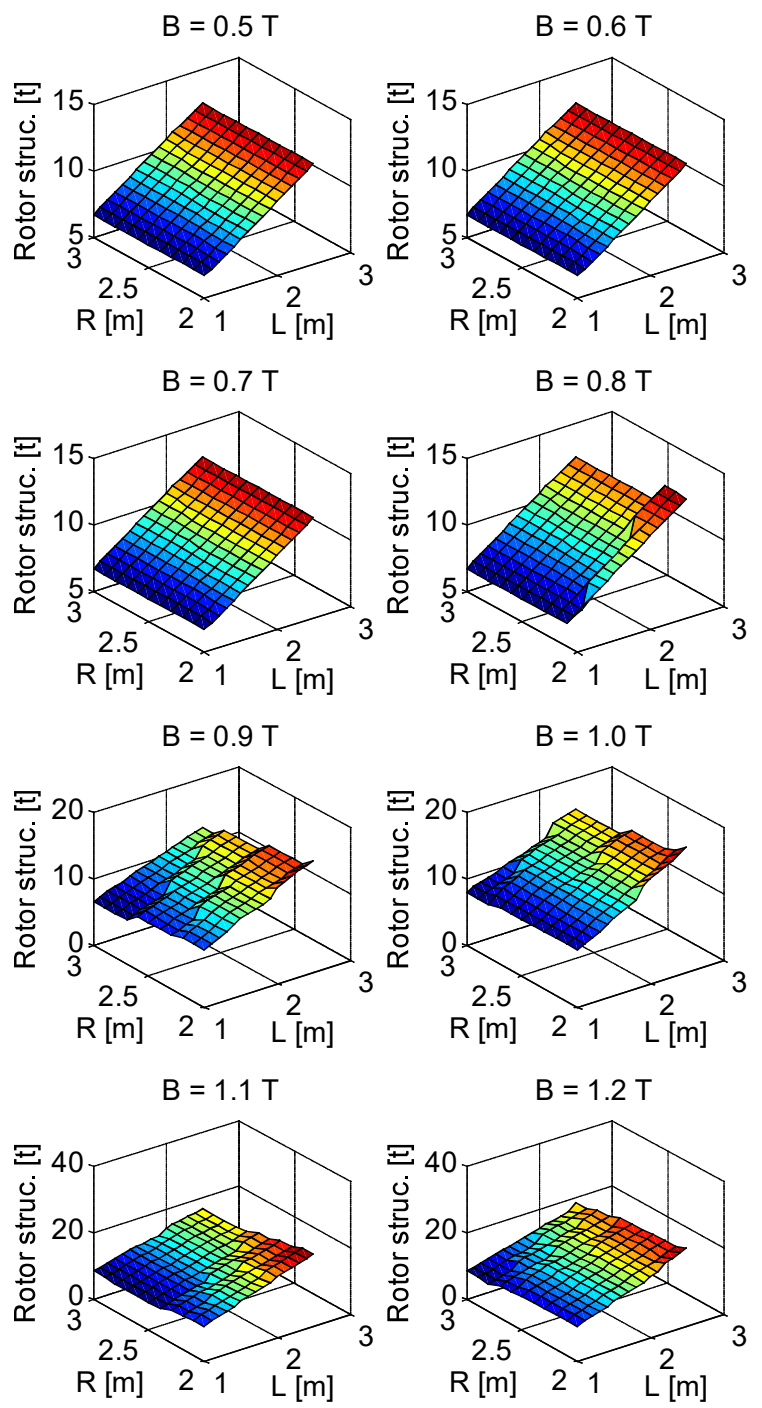

Figure 5: A set of structural mass results for the rotor, where a total air gap deformation of $10.0 \%$ is allowed

\subsection{Example Designs, with and without Structural Mass}

The results from two optimal design runs for a $3 \mathrm{MW}$, $15 \mathrm{rpm}$ direct-drive wind turbine are shown in Table 3. The objective function is minimization of the cost of energy (COE). For design (1) the structural mass is ignored, while in design two it is included.

Inclusion of the structural mass has resulted in an increase in COE of about 1.5 euro cents per $\mathrm{kWh}$. While design (1) converged upon a higher electrical loading and lower air gap flux density, for design (2), the inclusion of structural mass encouraged a lower air gap flux density and a higher electrical loading.

Table 3: Results for a pair of optimal designs for a 3MW $15 \mathrm{rpm}$ generator, first without incorporating structural mass, and second with

\begin{tabular}{llllll}
\hline & $\hat{B}[\mathrm{~T}]$ & $A[\mathrm{kA} / \mathrm{m}]$ & $r_{s}[\mathrm{~m}]$ & $l_{s}[\mathrm{~m}]$ & $\mathrm{COE}[€ / \mathrm{kWh}]$ \\
\hline$(1)$ & 0.79 & 80.0 & 2.3 & 1.3 & 0.346 \\
$(2)$ & 0.69 & 70.0 & 2.3 & 1.7 & 0.359 \\
\hline
\end{tabular}

\section{Conclusion}

Creation of a structural mass lookup table for incorporation into a direct-drive wind turbine generator design process has been demonstrated. The method consists of taking a planned design space and presimulating all anticipated machine geometries. It has been shown that the cost of energy is affected by inclusion of the structural mass, and that this also can influence the optimal design of a direct-drive generator.

\section{Acknowledgement}

The authors would like to thank DONG Energy for their support of the $\mathrm{PhD}$ project "Wind Turbine Generators with Reduced Reliance on Rare Earth Metals."

\section{References}

[1] A. McDonald, M. Mueller, \& H. Polinder, "Structural mass in direct-drive permanent magnet electrical generators", Renewable Power Generation, IET, 2008, 2, 3-15

[2] A. Grauers, A., "Design of direct-driven permanent-magnet generators for wind turbines", School of Electrical and Computer Engineering, Chalmers University of Technology, 1996

[3] A. Zavvos, A. McDonald, \& M. Mueller, "Electromagnetic and mechanical optimisation of direct-drive generators for large wind turbines", 5th IET International Conference on Power Electronics, Machines and Drives (PEMD 2010), 2010, 1-6

[4] A.S. McDonald, "Structural analysis of low speed, high torque electrical generators for direct drive renewable energy converters", University of Edinburgh, 2008

[5] R.S. Semken, M. Polikarpova, P. Roytta, J. Alexandrova, J. Pyrhonen, J. Nerg, A. Mikkola, J. Backman, "Direct-drive permanent magnet generators for high-power wind turbines: Benefits and limiting factors", Renewable Power Generation, IET, IET, 2012, 6, 1-8 